\title{
Orthotropic rotation-free basic thin shell triangle
}

\author{
J. G. Valdés · E. Oñate
}

Received: 14 July 2008 / Accepted: 21 January 2009 / Published online: 3 March 2009

(C) Springer-Verlag 2009

\begin{abstract}
A methodology for the geometrically nonlinear analysis of orthotropic shells using a rotation-free shell triangular element is developed. The method is based on the computation of the strain and stress fields in the principal fiber orientation of the material. Details of the definition of the fiber orientation in a mesh of triangles and of the general formulation of the orthotropic rotation-free element are given. The accuracy of the formulation is demonstrated in examples of application.
\end{abstract}

Keywords Rotation-free shell triangle - Thin shell . Orthotropic material · Principal fiber orientation

\section{Introduction}

Shells are used in many structural fields such as civil, mechanical, naval and aerospace engineering. A formulation based on thin-shell theory can be used to analyze many shell structures of practical interest. Thin-shell theory is also the basis for developing rotation-free finite elements which use the displacements as the only degrees of freedom (DOFs). This allow us to compute structures with less DOF and therefore less computational effort is required.

The idea of using the displacements as the only nodal variables for plate and shell analysis is not new and many attempts to derive this class of elements are found in the finite difference literature, i.e. Ugural [1]. The well-known difficulties of

\section{J. G. Valdés $(\varangle) \cdot$ E. Oñate}

International Center for Numerical Methods in Engineering (CIMNE), Technical University of Catalunya (UPC), Gran Capitan s/n, Campus Nord, 08034 Barcelona, Spain

e-mail: valdes@cimne.upc.edu

E. Oñate

e-mail: onate@ cimne.upc.edu finite difference analysis with boundary conditions and the problems for dealing with non-orthogonal or unstructured grids limited their progress. One of the first successes to use finite elements was due to Nay and Utku [2] who proposed a rotation-free thin plate triangle using a least-square quadratic approximation to describe the deflection field within the patch surrounding a node in terms of the deflections of the patch nodes. Later, Barnes [3] presented a method for deriving a three-noded triangle with the nodal deflections as the only DOFs based on the computation of the curvatures in terms of the nodal rotations at the middle-side points from the nodal deflections of adjacent elements. This method was exploited by Hampshire et al. [4] assuming that the elements are hinged together at their common boundaries and the bending stiffness is represented by torsional springs resisting rotations about the hinge line. Oñate and Cervera [5] presented a general procedure based on finite volume concepts for deriving thin plate elements of triangular and quadrilateral shapes with the nodal deflections as the only degree of freedom and proposed the first version of the so-called rotation-free basic plate triangle (BPT). Brunet and Sabourin [6] proposed a different approach to compute the constant curvature field within each triangle in terms of the six-node displacement of a macro-element. This triangular element was successfully applied to nonlinear shell analysis using an explicit dynamic approach. Oñate and Zárate [7] extended the rotation-free plate elements of Oñate and Cervera [5] developing new triangular elements, including a rotation-free basic shell triangle (BST). The BST element applied to large deformations with an explicit dynamic procedure was presented by Cendoya et al. [8]. Rojek et al. [9] applied the BST element to metal forming processes. The formulation of the BST element for large strain plasticity was introduced by Flores and Oñate [10]. Improvements of the BST element using an assumed strain approach were reported by Flores and Oñate 
[11]. A survey of recent developments of the BST element are reported in [12]. An alternative method for developing rotation-free shell elements using a subdivision of surfaces for thin-shell analysis was introduced by Cirak et al. [13] for small strains, and Cirak and Ortiz [14] for large deformations. Recent developments in the field including a survey of the performance of rotation-free shell triangles are reported in [15-18].

In this paper we present an extension of the BST element for the geometrically nonlinear analysis of orthotropic shells. The method is based on the principal fiber orientation of the material. It is shown that the fiber orientation strategy also allows to analyze pre-stressed shell structures. The accuracy of the orthotropic BST element is demonstrated in two examples of application.

\section{Thin shell formulation}

A finite deformation shell formulation is presented. Here Greek indices take on values of 1 and 2 while lower latin indices take on values of 1,2 and 3 . The position vector $\widetilde{\mathbf{R}}$ in the reference configuration $\Omega_{0}$ is defined by the independent
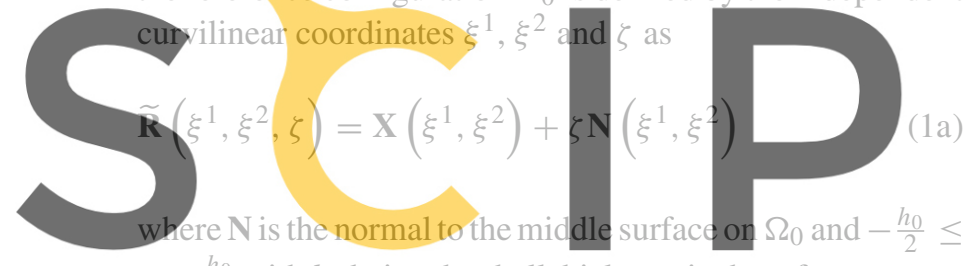

$\zeta \leq \frac{h_{0}}{2}$ with $h_{0}$ being the shell thickness in the reference con-

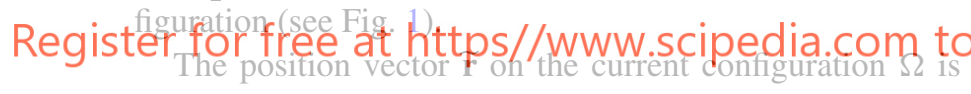
given by

$\widetilde{\mathbf{r}}\left(\xi^{1}, \xi^{2}, \zeta\right)=\mathbf{x}\left(\xi^{1}, \xi^{2}\right)+\zeta \mathbf{n}\left(\xi^{1}, \xi^{2}\right)$

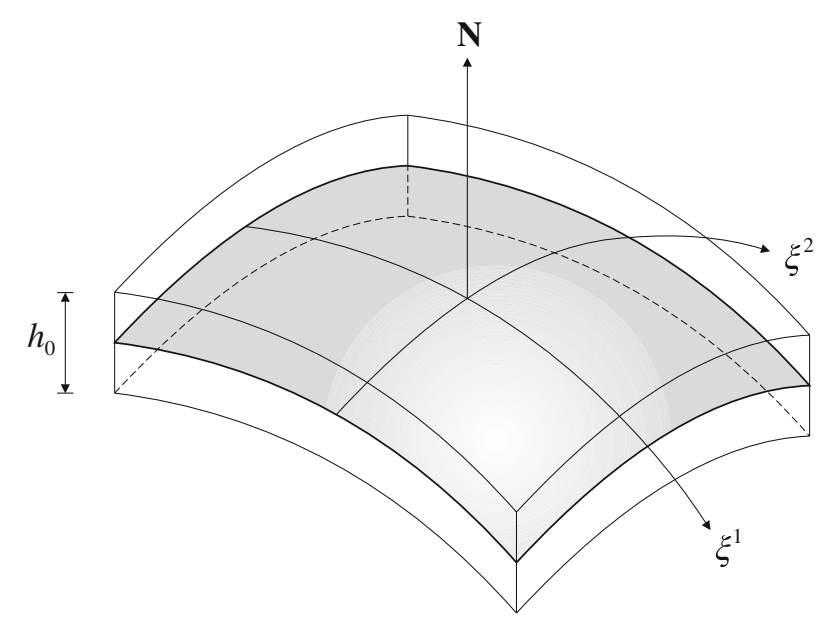

Fig. 1 Shell middle surface where $\mathbf{n}$ is the normal to the middle surface on $\Omega$. The relationship between the position vectors of the middle surface is

$\mathbf{x}=\mathbf{X}+\mathbf{u}$

where $\mathbf{u}$ is the displacement vector of the points on the middle surface.

Note that the finite thickness stretch parameter is not included in Eq. (1b) to avoid Poisson locking. The finite thickness stretch plays an important role in problems involving finite membrane strains or contact, as shown in [19-21]. In this work a Saint Venant-Kirchhoff material model is used and small strains are assumed which allows neglecting the thickness stretch effect.

The convected covariant base vectors of the curvilinear coordinate system on $\Omega_{0}$ are defined by

$$
\begin{aligned}
& \widetilde{\mathbf{G}}_{\alpha}=\frac{\partial \widetilde{\mathbf{R}}}{\partial \xi^{\alpha}}=\frac{\partial \mathbf{X}}{\partial \xi^{\alpha}}+\zeta \frac{\partial \mathbf{N}}{\partial \xi \alpha}=\mathbf{G}_{\alpha}+\zeta \mathbf{N}, \alpha \\
& \widetilde{\mathbf{G}}_{3}=\frac{\partial \widetilde{\mathbb{R}}}{\partial \zeta}=\mathbf{N}
\end{aligned}
$$

where $\mathbf{G}_{\alpha}$ are the middle surface basis vectors in the reference configuration. The convected covariant base vectors on
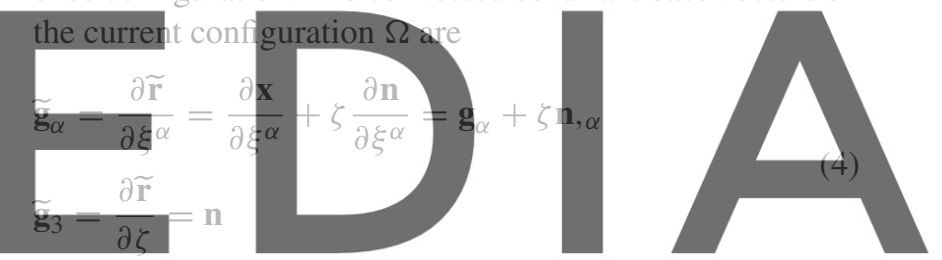

where $\mathrm{g}_{\alpha}$ are the middle surface basis vectors in the current

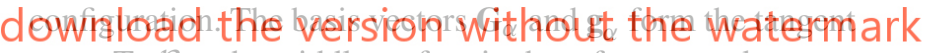
space $\mathrm{T}_{X} \mathcal{B}$ to the middle surface in the reference and current configurations, respectively and in general they are neither unit vectors nor orthogonal to each other, shown in Fig. 2. For finite element problems, a tangent space is built at every gauss point of the element. In this work, three-node triangles are used and therefore the tangent space coincides with the element plane, and only one gauss point is needed per element.

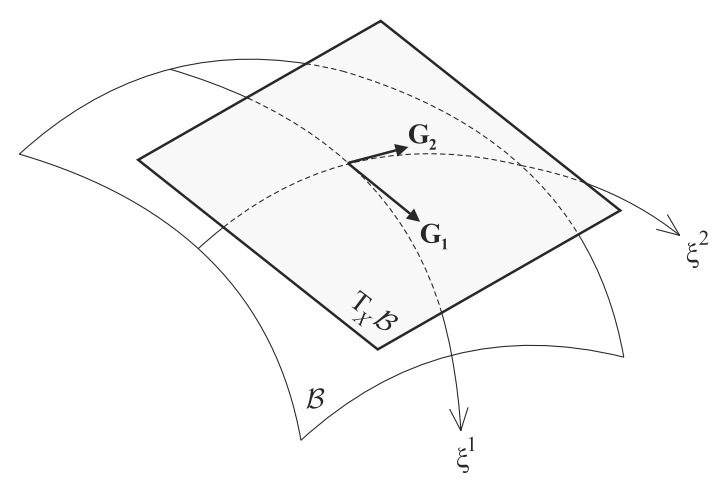

Fig. 2 Covariant base vectors forming a tangent plane 
The convected contravariant base vectors follow from the relations

$\widetilde{\mathbf{G}}^{i} \cdot \widetilde{\mathbf{G}}_{j}=\delta_{j}^{i}, \quad \widetilde{\mathbf{g}}^{i} \cdot \widetilde{\mathbf{g}}_{j}=\delta_{j}^{i}$

where $\delta_{j}^{i}$ is the Kronecker delta. The covariant metric tensors in both configurations are

$\widetilde{G}_{i j}=\widetilde{\mathbf{G}}_{i} \cdot \widetilde{\mathbf{G}}_{j}, \quad \widetilde{g}_{i j}=\widetilde{\mathbf{g}}_{i} \cdot \widetilde{\mathbf{g}}_{j}$

The components of the Green-Lagrange strain tensor are defined as the difference between the covariant metric tensors on the current and reference configurations of the shell yielding

$E_{i j}=\frac{1}{2}\left(\widetilde{g}_{i j}-\widetilde{G}_{i j}\right)$

The Green-Lagrange strain tensor can be written as

$E_{i j}=\epsilon_{i j}+\zeta \kappa_{i j}+\zeta^{2} \gamma_{i j}$

where the non-zero components of the above expression are given by

$\epsilon_{\alpha \beta}=\frac{1}{2}\left(\mathbf{g}_{\alpha} \cdot \mathbf{g}_{\beta}-\mathbf{G}_{\alpha} \cdot \mathbf{G}_{\beta}\right), \quad \epsilon_{\alpha 3}=\frac{1}{2}\left(\mathrm{~g}_{\alpha} \cdot \mathbf{n}-\mathbf{G}_{\alpha} \cdot \mathbf{N}\right.$

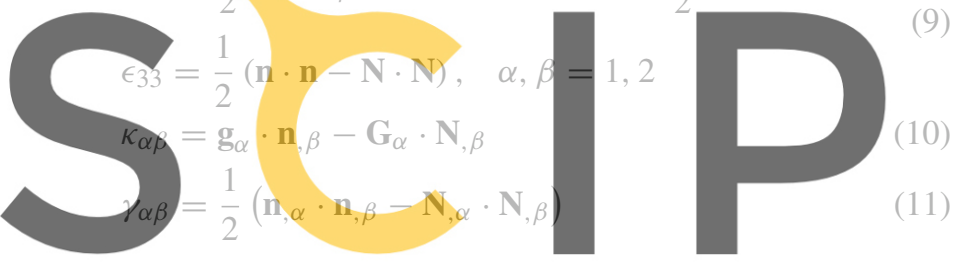

This work is intended for the Kirchhoff-Love theory of thin Register for free at hittos fomed wirectipn goincides with the unit normal to the current middle surface. Therefore the values $\epsilon_{\alpha 3}$ and $\epsilon_{33}$ vanish identically and values of $\zeta^{2}$ can be neglected for thin shells. This constraint yields the components of the Green-Lagrange strain tensor to be deduced from the deformation of the middle surface of the shell as

$E_{\alpha \beta}=\epsilon_{\alpha \beta}+\zeta \kappa_{\alpha \beta}=E_{\alpha \beta}^{\mathrm{memb}}+\zeta E_{\alpha \beta}^{\mathrm{bend}}$

where $\epsilon_{\alpha \beta}$ measures membrane strains. For convenience of the discretization explained ahead, the bending strains are written as

$\kappa_{\alpha \beta}=\mathbf{G}_{\alpha, \beta} \cdot \mathbf{N}-\mathbf{g}_{\alpha, \beta} \cdot \mathbf{n}=K_{\alpha \beta}-k_{\alpha \beta}$

where Eq. 13 is derived from Eq. 10 and the following equation is satisfied

$\left(\mathbf{g}_{\alpha} \cdot \mathbf{n}\right)_{\beta}=\mathbf{g}_{\alpha} \cdot \mathbf{n}_{, \beta}+\mathbf{g}_{\alpha, \beta} \cdot \mathbf{n}=0$

The same applies for $\left(\mathbf{G}_{\alpha} \cdot \mathbf{N}\right)_{, \beta}$. The variation of the GreenLagrange strain tensor is given by the variation of Eq. 12 yielding

$\delta E_{\alpha \beta}=\delta E_{\alpha \beta}^{\mathrm{memb}}+\zeta \delta E_{\alpha \beta}^{\mathrm{bend}}$ with an appropriate constitutive equation to relate stresses and strains, the virtual internal work is expressed as

$\delta \mathcal{W}^{\text {int }}=\int_{\Omega_{0}} \int_{-\frac{h}{2}}^{+\frac{h}{2}} \delta E_{\alpha \beta} S^{\alpha \beta} d \zeta d \bar{\Omega}_{0}$

where $\bar{\Omega}_{0}$ is the middle surface domain. The expression for the virtual work of the external loads is the classical one for shells [22].

\section{Fiber orientation}

The idea for the fiber orientation approach comes from the manufacturing process of membrane structures manufactured with orthotropic or composite materials. Here a reference principal fiber direction is needed to perform an analysis with finite elements. Even for isotropic materials, a reference direction is needed if the membrane structure has an initial prestressed field. With the methodology proposed here, a prestressed field for orthotropic materials is also possible.

Another important aspect of the fiber orientation comes

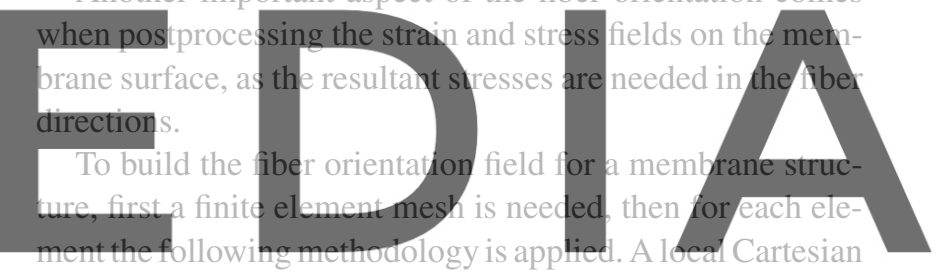

base system is defined from the covariant base vectors as

download the version without the watermark

$\mathrm{e}_{1}^{\mathrm{loc}}=\frac{}{\left\|\mathrm{G}_{1}\right\|}, \quad \mathrm{e}_{3}^{\mathrm{loc}}=\mathrm{N}=\frac{\mathrm{G}_{1} \times \mathrm{G}_{2} \|}{\|}$

$\mathbf{e}_{2}^{\text {loc }}=\mathbf{e}_{3}^{\text {loc }} \times \mathbf{e}_{1}^{\text {loc }}$

If a curved membrane structure is meshed with finite elements, the local Cartesian axes of each element generally have different orientations, even for structured meshes as shown in Fig. 3.

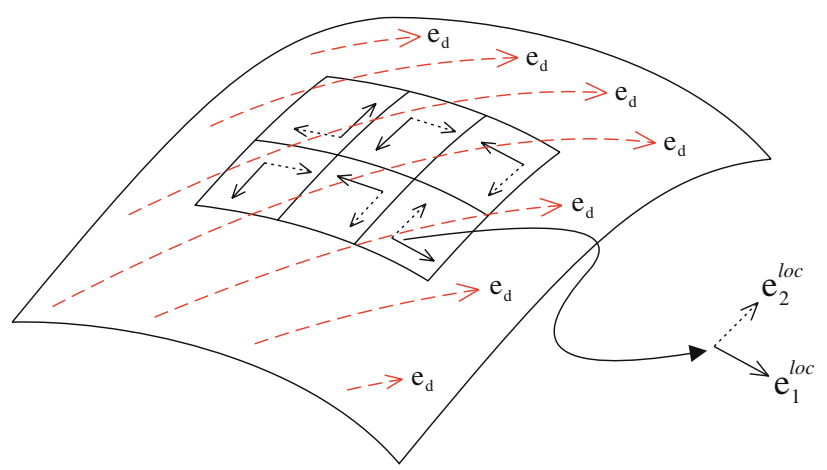

Fig. 3 Principal fiber direction and local base system 
Fig. 4 Principal fiber direction for a finite element

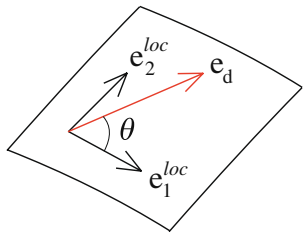

If dashed-lines $\mathbf{e}_{\mathrm{d}}$ are the reference principal fibers orientation or the orthotropy direction for the material, then an angle $\theta$ is needed to rotate each local Cartesian base system $\mathbf{e}_{i}^{\text {loc }}$ in order to apply the orthotropic constitutive equation correctly (or the prestressed field). Suppose that $\mathbf{e}_{\mathrm{d}}$ is a given vector that defines the principal fiber orientation for a finite element and it lies on the tangent space of the element, as shown in Fig. 4. For curved elements, vector $\mathbf{e}_{\mathrm{d}}$ is obtained by taking the proiection of the fiber direction onto the tangent space of the element. This principal fiber direction is always known at least for one finite element and it is given by the manufacturing process of the structure.

To assign correctly the principal fiber direction from a known source element to the whole mesh, first the adjacent elements of the source element must be identified (see Fig. 5a). Next the tangent space $T_{X} \mathcal{B}^{S}$ for the source element is built, together with its norma

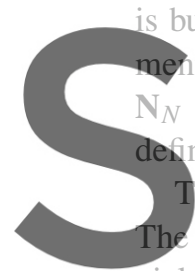

its tangent space $\mathrm{T}_{X} \mathcal{B}^{N}$ are built. The intersection line es the common vector ( hen a new Cartesian base syste rields $\mathbf{Y}_{S}$. This new vector $\mathbf{Y}_{S}$ must point towards inside of the source element, as shown in Fig. 6.

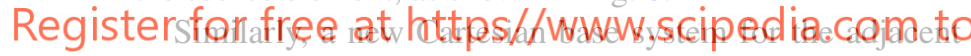
element is built. The cross product between the normal $\mathrm{N}_{N}$ and the vector given by the intersection line $\mathbf{X}_{N}$ yields $\mathbf{Y}_{N}$, where $\mathbf{X}_{N}=-\mathbf{X}_{S}$. Vector $\mathbf{Y}_{N}$ must point towards inside of the adjacent element. Note that vectors $\mathbf{X}_{S}$ and $\mathbf{Y}_{S}$ belong to the tangent space of the source element, while vectors $\mathbf{X}_{N}$ and $\mathbf{Y}_{N}$ belong to the tangent space of the adjacent element.

Once both local Cartesian base systems are defined, the next step is to choose an arbitrary point $a$ on the axis $\mathbf{X}_{S}$. Now over point $a$ the principal fiber direction vector $\mathbf{e}_{\mathrm{d}}$ is built and its intersection over the axis $\mathbf{Y}_{S}$ will be point $b$, as shown in Fig. 7.

Finally, to transfer the principal fiber direction to the adjacent element, the distance from the origin $o$ to point $a$ must be

Fig. 5 Assignment of principal fiber direction to adjacent elements the same that the distance from the origin $o$ to point $c$ located on the axis $\mathbf{X}_{N}$. Also the distance from the origin $o$ to point $b$ must be the same as from the origin $o$ to point $d$ over the axis $\mathbf{Y}_{N}$. Then the vector from point $c$ to point $d$ is built. This vector will be the reference principal fiber direction in the neighbor element, as shown in Fig. 7.

This procedure of transferring the principal fiber direction from a source element to an adjacent one is repeated for all the elements adjacent to the source element, as shown in Fig. 5. Once all adjacent elements have a principal fiber direction defined, the procedure is repeated for the whole mesh until every element has a principal fiber direction $\mathbf{e}_{\mathrm{d}}$ assigned to it. A finite element mesh with all its elements with a principal fiber direction assigned is shown in Fig. 8.

The next step is to define for each element an angle $\theta$ needed to rotate each local Cartesian system to the fiber system (Fig. 4). Angle $\theta$ is found from

$\sin \theta=-\mathbf{e}_{\mathrm{d}} \cdot \mathbf{e}_{2}^{\mathrm{loc}}, \quad \cos \theta=\mathbf{e}_{\mathrm{d}} \cdot \mathbf{e}_{1}^{\mathrm{loc}}$

and

$\theta=\tan ^{-1}\left(\frac{\sin \theta}{\cos \theta}\right)$

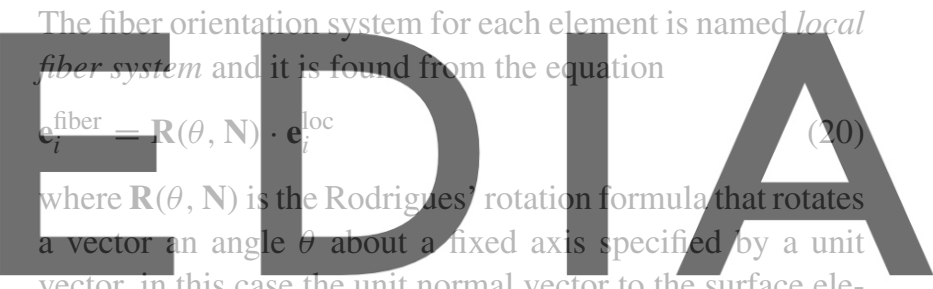

vector, in this case the unit normal vector to the surface element $\mathbf{N}$.

downd aad ethenversion theithous thenevatermark entation is shown in Fig. 9. Details of the implementation of the fiber orientation methodology can be found in Valdés [23].

\section{Finite element discretization for shells}

The finite element discretization is given for the total Lagrangian formulation. In this work the BST element developed in [10-12] is extended to account for the principal fiber direction and perform the analysis with initially prestressed fields or orthotropic materials. We note that the BST element is free of rotational DOFs. Therefore the only

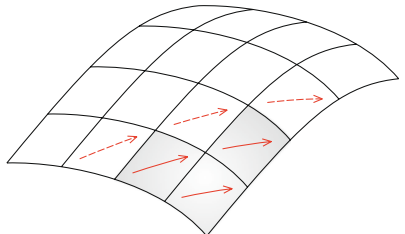

(b)

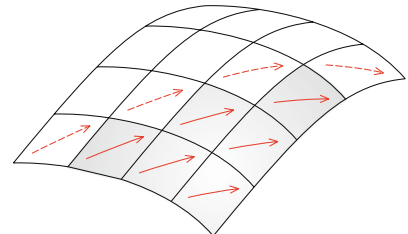

(c) 


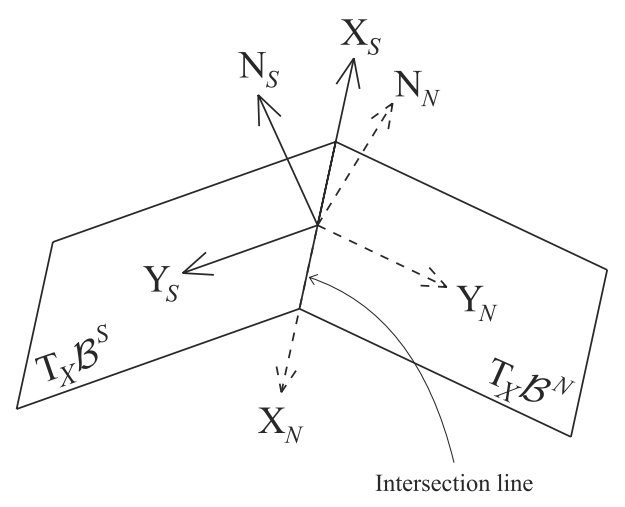

Fig. 6 Local Cartesian systems for tangent spaces
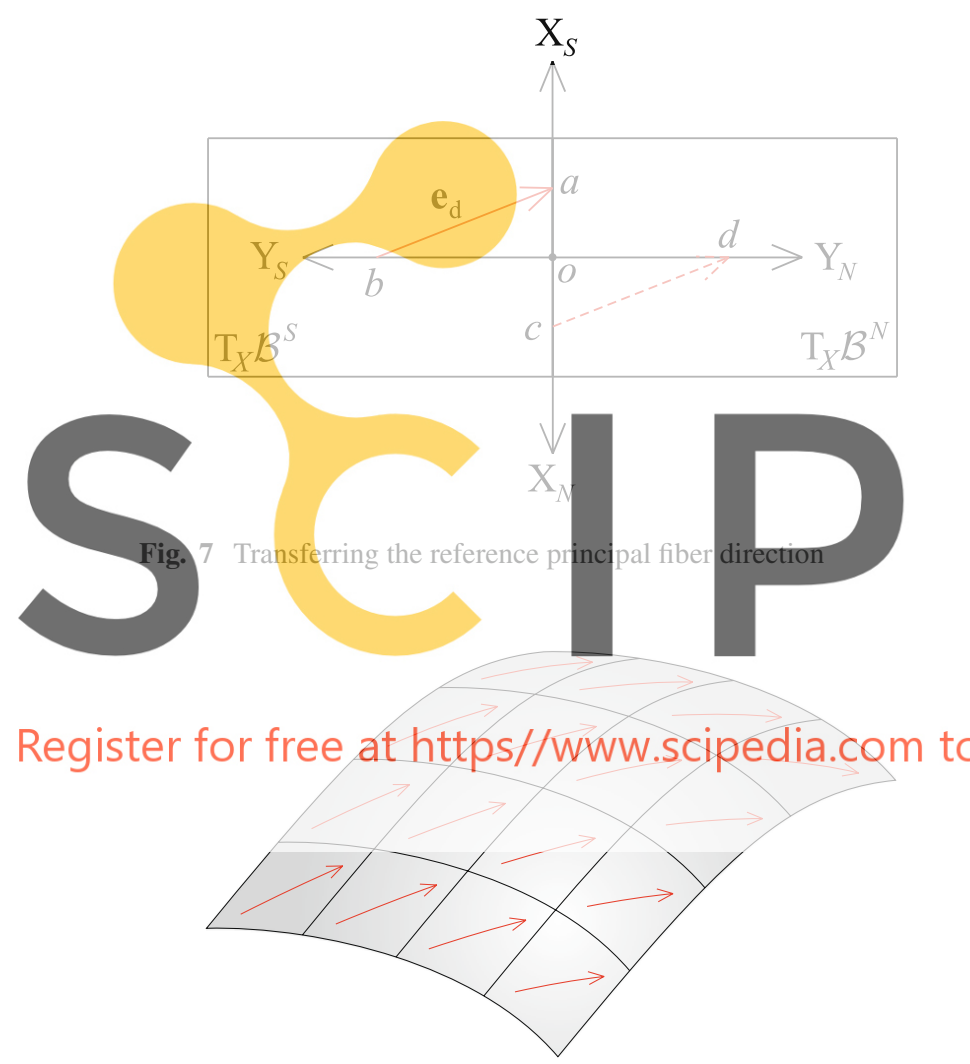

Fig. 8 Principal fibers direction of a mesh

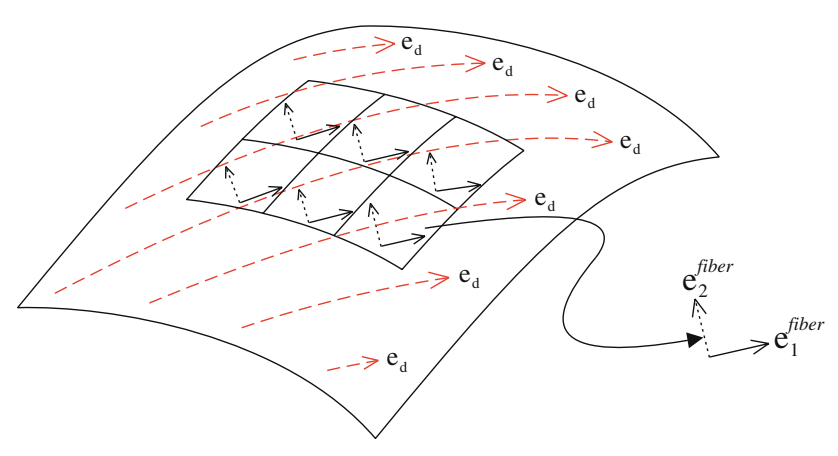

Fig. 9 Local fiber system

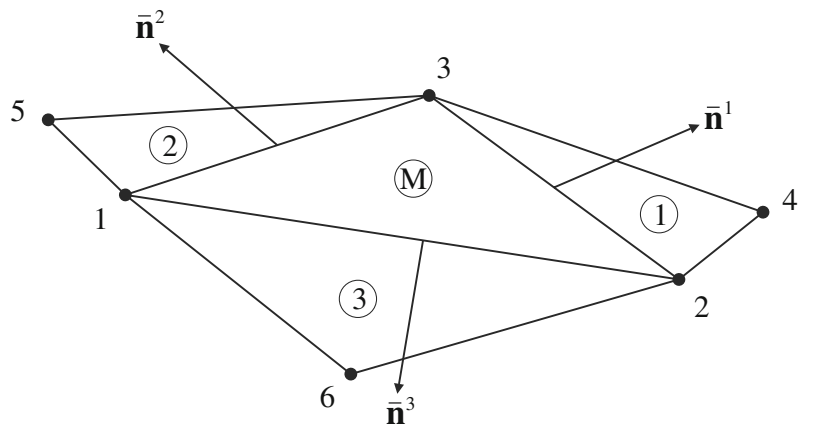

Fig. 10 Four element patch for computation of bending strains

Table 1 Patch connectivities for the BST element

\begin{tabular}{llll}
\hline Element & \multicolumn{2}{l}{ Connectivities } & \\
\cline { 2 - 4 } & Node 1 & Node 2 & Node 3 \\
\hline (M) & 1 & 2 & 3 \\
$(1)$ & 4 & 3 & 2 \\
$(2)$ & 5 & 1 & 3 \\
$(3)$ & 6 & 2 & 1 \\
\hline
\end{tabular}

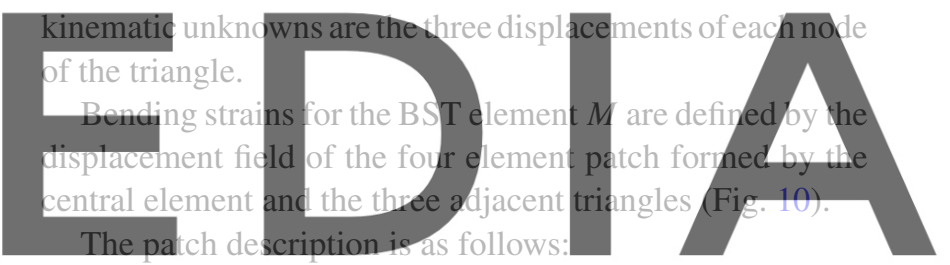

\section{dowhidoand ntmeeverissiona widtlpout the watermark \\ - Nodes of the main element (M) are numbered locally as 1,2 and 3 .}

- Main element sides are defined by its local node opposite to the side.

- Adjacent elements are numbered with the number associated to the common side 1,2 and 3 .

- The remaining nodes of the patch are numbered locally as 4,5 , and 6 corresponding to nodes on adjacent elements (1), (2) and (3) respectively.

Connectivities for the path are defined in Table 1.

In this work, the local coordinate system is given by the local fiber system, defined in Sect. 3. Then the local coordinate system for each finite element is given by unit vectors $\mathbf{e}_{1}^{\mathrm{fib}}, \mathbf{e}_{2}^{\mathrm{fib}}$ and the normal $\mathbf{e}_{3}^{\mathrm{fib}}$.

\subsection{Computation of the membrane strains}

The computation of the membrane strain follows the standard approach for the constant strain triangle as detailed in Valdés [23]. 
The covariant base vectors of the curvilinear coordinates in the reference configuration $\Omega_{0}$ can be approximated by

$\mathbf{G}_{\alpha}=\sum_{I=1}^{n_{\text {node }}} N_{I, \alpha} \mathbf{X}_{I}$

where

$N_{I, \alpha}=\frac{\partial N_{I}\left(\xi^{1}, \xi^{2}\right)}{\partial \xi^{\alpha}}$

being $N_{I}$ the element shape functions. Following the same procedure, the covariant base vectors of the curvilinear coordinates in the current configuration $\Omega$ reads

$\mathbf{g}_{\alpha}=\sum_{I=1}^{n_{\text {node }}} N_{I, \alpha} \mathbf{x}_{I}(t)$

with these quantities known, the covariant components of the metric tensors $G_{\alpha \beta}$ and $g_{\alpha \beta}$ are found. Then the components of the Green-Lagrange strain tensor for the membrane part are computed with

$\epsilon_{\alpha \beta}=\frac{1}{2}\left(g_{\alpha \beta}-G_{\alpha \beta}\right)$
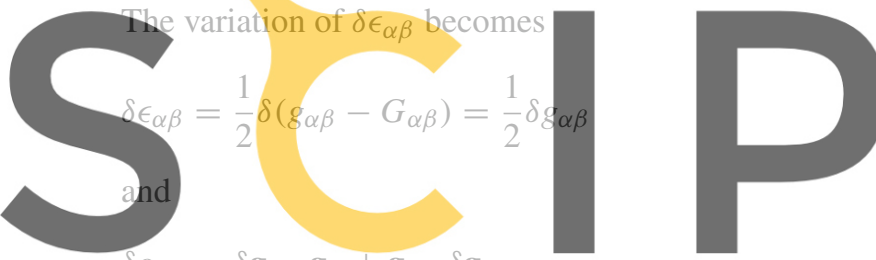

$\delta g_{\alpha \beta}=\delta \mathbf{g}_{\alpha} \cdot \mathrm{g}_{\beta}+\mathrm{g}_{\alpha} \cdot \delta \mathrm{g}_{\beta}$

(26)

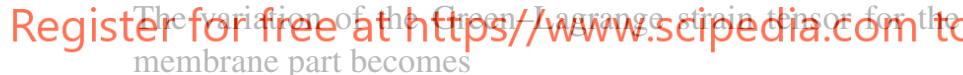

$$
\begin{aligned}
2 \delta \epsilon_{\alpha \beta}= & \sum_{I=1}^{n_{\text {node }}} N_{I, \alpha} \delta u_{i I} \cdot \sum_{J=1}^{n_{\text {node }}} N_{J, \beta} x_{i J} \\
& +\sum_{J=1}^{n_{\text {node }}} N_{J, \alpha} x_{i J} \cdot \sum_{I=1}^{n_{\text {node }}} N_{I, \beta} \delta u_{i I}
\end{aligned}
$$

where $\delta u_{i}$ are the virtual displacements $(i=1,3)$. The virtual internal work due to membrane effects is (see Eq. 16)

$$
\begin{aligned}
2 \delta \mathcal{W}^{\text {int }}= & h \int_{\Omega_{0}}\left[\sum_{I=1}^{n_{\text {node }}} N_{I, \alpha} \delta u_{i I} \cdot \sum_{J=1}^{n_{\text {node }}} N_{J, \beta} x_{i J} S_{\text {memb }}^{\alpha \beta}\right. \\
& \left.+\sum_{J=1}^{n_{\text {node }}} N_{J, \alpha} x_{i J} \cdot \sum_{I=1}^{n_{\text {node }}} N_{I, \beta} \delta u_{i I} S_{\text {memb }}^{\alpha \beta}\right] d \bar{\Omega}_{0}
\end{aligned}
$$

Equation (28) can also be expressed as

$\delta \mathcal{W}^{\text {int }}=\sum_{I=1}^{n_{\text {node }}} \delta u_{i I} f_{i I}^{\text {memb }}, \quad i=1,3$
Substituting Eq. (28) into Eq. (29), the internal membrane forces for a particular direction $i$ and node $I$ can be expressed as

$$
f_{i I}^{\mathrm{memb}}=h \int_{\bar{\Omega}_{0}} \mathcal{B}_{\alpha \beta i I}^{\mathrm{memb}} S_{\mathrm{memb}}^{\alpha \beta} d \bar{\Omega}_{0}
$$

where the strain-displacement tensor in curvilinear coordinates is given by

$\mathcal{B}_{\alpha \beta i I}^{\mathrm{memb}}=\frac{1}{2}\left(N_{I, \alpha} x_{i, \beta}^{h}+N_{I, \beta} x_{i, \alpha}^{h}\right)$

where the isoparametric interpolation [22]

$x_{i, \alpha}^{h}=\sum_{J=1}^{n_{\text {node }}} N_{J, \alpha} x_{i J}$

has been used. The expression of the internal forces in curvilinear coordinates using Voigt notation is
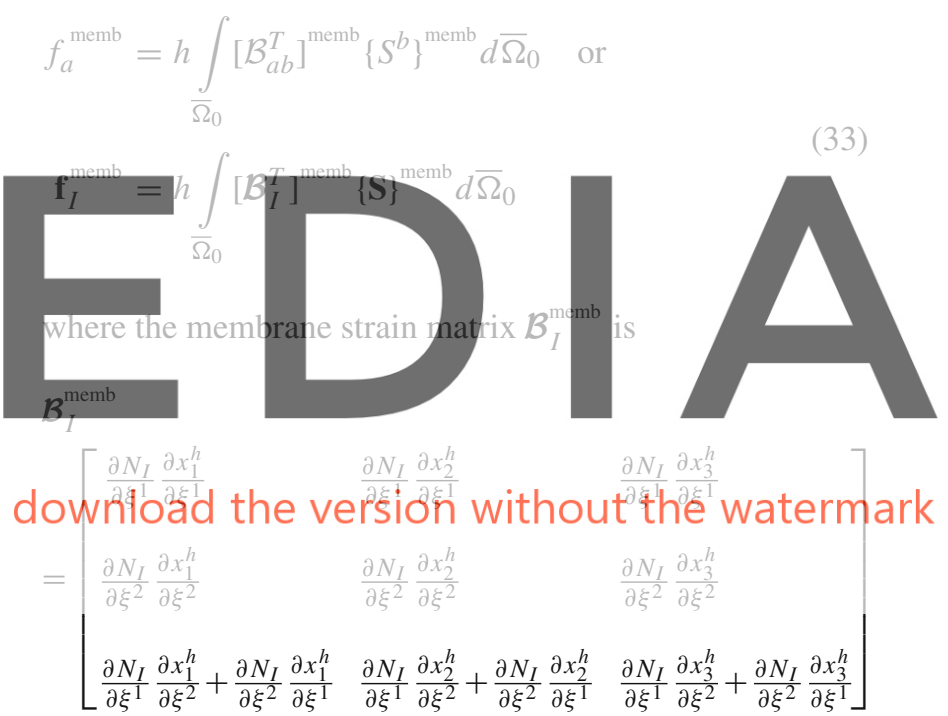

Finally, the membrane strain matrix is rotated from curvilinear to Cartesian coordinates. Then using the fiber orientation methodology, it is rotated again to fiber direction yielding $\mathbf{B}_{I}^{\mathrm{memb}}$.

\subsection{Computation of the bending strains}

The computation of the bending strains for the rotation-free BST element requires a more detailed explanation which is presented next. The main difference between the present BST formulation and the BST formulation of Flores and Oñate [10] is the local coordinate system definition. While Flores and Oñate define direction $\xi^{1}$ along nodes 1 and 2, in this work $\xi^{1}$ follows the principal fiber direction. This change is what allow us to analyze prestressed shell structures and/or 
orthotropic shells, and for this reason the formulation is presented in detail. The bending part of Eq. (16) is given by,

$\delta \mathcal{W}^{\text {int }}=\int_{\bar{\Omega}_{0}}^{+\frac{h}{2}} \int_{-\frac{h}{2}}^{\text {bend }} \delta E_{\alpha \beta}^{\alpha \beta} S_{\text {bend }} d \zeta d \bar{\Omega}_{0}$

and the values of $E_{\alpha \beta}^{\text {bend }}$ and $\delta E_{\alpha \beta}^{\text {bend }}$ are needed to evaluate the internal forces. The bending strains at the current configuration are expressed by (Eq. 13)

$k_{\alpha \beta}=\mathbf{g}_{\alpha, \beta} \cdot \mathbf{n}$

which can be written in the form

$k_{\alpha \beta}=\frac{1}{A_{0}} \int_{\bar{\Omega}} \mathbf{g}_{\alpha, \beta} d \bar{\Omega}_{0} \cdot \mathbf{n}$

Applying the divergence theorem Eq. (37) yields

$k_{\alpha \beta}=\frac{1}{A_{0}} \int_{\bar{\Gamma}_{0}} \bar{n}_{\beta} \mathbf{g}_{\alpha} d \bar{\Gamma}_{0} \cdot \mathbf{n}$

where $\bar{n}_{\beta}$ are the components of the normal to the boundary, laying on the plane of element $M$ (Fig. 10). Since the bound-

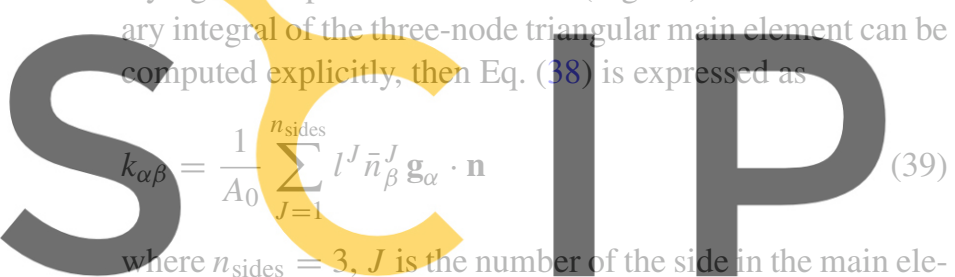
ment, $l^{J}$ is the length of side $J$ and $\bar{n}_{\beta}^{J}$ are the components Register for free at httpsy ww wide sof the main element. ear coordinates to the local fiber system, so the same local coordinate system is used for the whole patch.

Discretization of $\mathbf{g}_{\alpha}$ can be written explicitly as

$\left[\begin{array}{l}\mathbf{g}_{1} \\ \mathbf{g}_{2}\end{array}\right]=\sum_{I=1}^{n_{\text {node }}}\left[\begin{array}{c}\frac{\partial N_{I}}{\partial \xi} \\ \frac{\partial N_{I}}{\partial \eta}\end{array}\right] \mathbf{x}_{I}(t)$

Since the formulation is derived in the local fiber system, the derivatives of the shape functions need to be transformed. The Jacobian transformation tensor $\mathbf{J}_{\xi}$ in the current configuration is defined as

$\mathbf{J}_{\xi}=\left[\begin{array}{lll}\mathbf{g}_{1} \cdot \mathbf{e}_{1}^{\mathrm{fib}} & \mathbf{g}_{2} \cdot \mathbf{e}_{1}^{\mathrm{fib}} \\ \mathbf{g}_{1} \cdot \mathbf{e}_{2}^{\mathrm{fib}} & \mathbf{g}_{2} \cdot \mathbf{e}_{2}^{\mathrm{fib}}\end{array}\right]$

and the Cartesian derivatives of the shape functions are obtained from

$\left[\begin{array}{c}\frac{\partial N_{I}}{\partial x} \\ \frac{\partial N_{I}}{\partial y}\end{array}\right]=\mathbf{J}_{\xi}^{-T}\left[\begin{array}{c}\frac{\partial N_{I}}{\partial \xi} \\ \frac{\partial N_{I}}{\partial \eta}\end{array}\right]$
Equation (40) can be written in Cartesian coordinates as

$\left[\begin{array}{c}\mathbf{x}_{, 1}^{h} \\ \mathbf{x}_{, 2}^{h}\end{array}\right]=\sum_{I=1}^{n_{\text {node }}}\left[\begin{array}{c}\frac{\partial N_{I}}{\partial x} \\ \frac{\partial N_{I}}{\partial y}\end{array}\right] \mathbf{x}_{I}(t)$

Using Voigt notation, the curvatures of Eq. (39) can be computed in the local fiber system as

$$
\left[\begin{array}{l}
k_{11} \\
k_{22} \\
k_{12}
\end{array}\right]=\frac{1}{A_{0}} \sum_{J=1}^{n_{\text {sides }}} l^{J}\left[\begin{array}{cc}
\bar{n}_{1}^{J} & 0 \\
0 & \bar{n}_{2}^{J} \\
\bar{n}_{2}^{J} & \bar{n}_{1}^{J}
\end{array}\right]\left[\begin{array}{l}
\mathbf{x}_{, 1}^{h} \cdot \mathbf{n} \\
\mathbf{x}_{, 2}^{h} \cdot \mathbf{n}
\end{array}\right]
$$

The constraints imposed by the Kirchhoff-Love theory of thin shells make the product $\mathbf{x}_{, \alpha}^{h} \cdot \mathbf{n}=0$. This problem is solved taking for $\mathbf{x}_{, \alpha}^{h}$ the average value between that of the main triangle and each one of the adjacent elements yielding

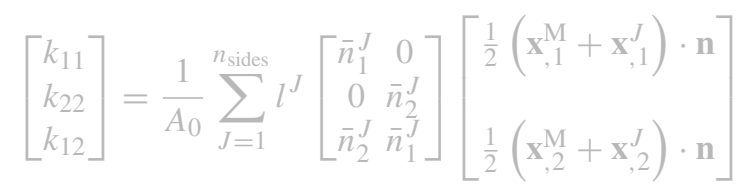

$(45)$

which can be simplified to

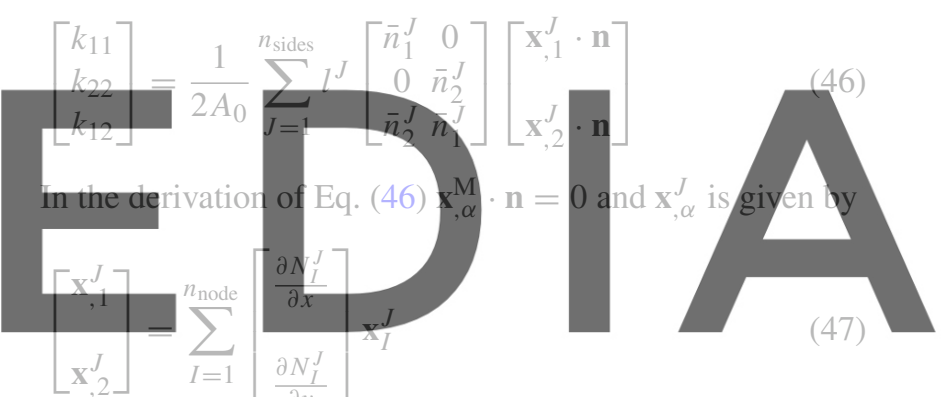

download the version without the watermark The same methodology is used to obtain the bending strains in the reference configuration. The bending strain tensor in Voigt notation is given by

$$
\{\mathbf{E}\}^{\text {bend }}=\left[\begin{array}{l}
\kappa_{11} \\
\kappa_{22} \\
\kappa_{12}
\end{array}\right]=\left[\begin{array}{l}
K_{11} \\
K_{22} \\
K_{12}
\end{array}\right]-\left[\begin{array}{l}
k_{11} \\
k_{22} \\
k_{12}
\end{array}\right]
$$

The variation of the bending strain tensor yields

$\delta\{\mathbf{E}\}^{\text {bend }}=\left[\begin{array}{l}\delta \kappa_{11} \\ \delta \kappa_{22} \\ \delta \kappa_{12}\end{array}\right]=-\left[\begin{array}{l}\delta k_{11} \\ \delta k_{22} \\ \delta k_{12}\end{array}\right]$

From Eq. (45)

$$
\left[\begin{array}{l}
\delta k_{11} \\
\delta k_{22} \\
\delta k_{12}
\end{array}\right]=\frac{1}{2 A_{0}} \sum_{J=1}^{n_{\text {sides }}} l^{J}\left[\begin{array}{cc}
\bar{n}_{1}^{J} & 0 \\
0 & \bar{n}_{2}^{J} \\
\bar{n}_{2}^{J} & \bar{n}_{1}^{J}
\end{array}\right]\left[\begin{array}{l}
\delta\left(\mathbf{x}_{, 1}^{J} \cdot \mathbf{n}\right) \\
\delta\left(\mathbf{x}_{, 2}^{J} \cdot \mathbf{n}\right)
\end{array}\right]
$$

The last term of the right-hand side of Eq. (50) can be expanded to become

$\delta\left(\mathbf{x}_{, \alpha}^{J} \cdot \mathbf{n}\right)=\delta \mathbf{x}_{, \alpha}^{J} \cdot \mathbf{n}+\mathbf{x}_{, \alpha}^{J} \cdot \delta \mathbf{n}$ 
The variation $\delta \mathbf{x}_{\alpha}^{J}$ yields

$\left[\begin{array}{l}\delta \mathbf{x}_{, 1}^{J} \\ \delta \mathbf{x}_{, 2}^{J}\end{array}\right]=\sum_{I=1}^{n_{\text {node }}}\left[\begin{array}{c}\frac{\partial N_{I}^{J}}{\partial x} \\ \frac{\partial N_{I}^{J}}{\partial y}\end{array}\right] \delta \mathbf{u}_{I}^{J}$

On the other hand, the variation $\delta \mathbf{n}$ becomes

$\delta \mathbf{n}=-\sum_{I=1}^{n_{\text {node }}}\left(\frac{\partial N_{I}}{\partial x} \tilde{\mathbf{x}}_{, 1}^{h}+\frac{\partial N_{I}}{\partial y} \tilde{\mathbf{x}}_{, 2}^{h}\right) \mathbf{n} \cdot \delta \mathbf{u}_{I}$

as detailed in Flores and Oñate [10] and Valdés [23]. Substituting Eqs. (52) and (53) into Eq. (51) leads to

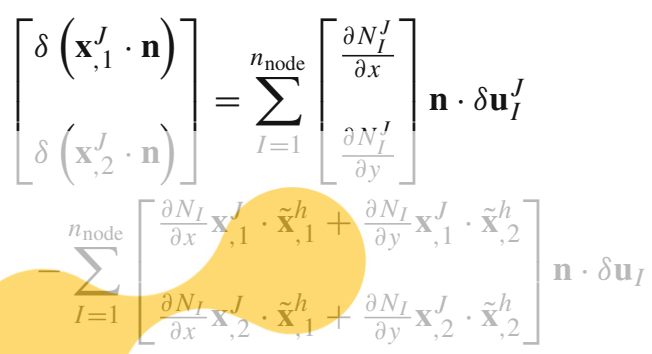

Now substituting Eq. (54) into Eq. (50), and the resulting equation into Eq. (49), the variation of the bending strain tensor can be written explicitly in Voigt notation as

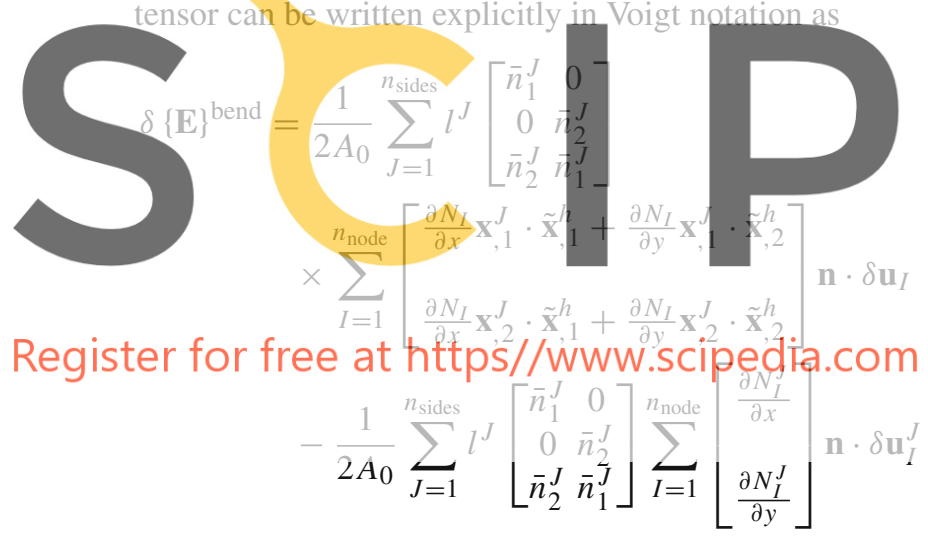

The variation of the bending strain tensor can be expressed in compact form as

$\delta\{\mathbf{E}\}^{\text {bend }}=[\mathbf{B}]^{\text {main }} \delta \mathbf{u}_{I}+[\mathbf{B}]^{\text {adj }} \delta \mathbf{u}_{I}^{J}$

where the expression of the bending matrix $[\mathbf{B}]^{\text {main }}$ for the main element and $[\mathbf{B}]^{\text {adj }}$ for the adjacent element is deduced from Eq. (55). The complete strain-displacement matrix for bending is given by

$[\mathbf{B}]^{\text {bend }}=[\mathbf{B}]^{\text {main }}+[\mathbf{B}]^{\text {adj }}$

\subsection{Stress resultants and internal forces}

The constitutive equation can be written for shell elements as

$$
\{\mathbf{S}\}=[\mathbf{C}] \cdot\{\mathbf{E}\}=[\mathbf{C}] \cdot\left(\{\mathbf{E}\}^{\text {memb }}+\zeta\{\mathbf{E}\}^{\text {bend }}\right)
$$

The virtual internal work Eq. (16) is expressed by

$$
\begin{aligned}
\delta \mathcal{W}^{\text {int }}= & \int_{\bar{\Omega}_{0}} \int_{-\frac{h}{2}}^{\frac{h}{2}}\left(\delta\{\mathbf{E}\}^{\text {memb }}+\zeta \delta\{\mathbf{E}\}^{\text {bend }}\right) \\
& \cdot[\mathbf{C}]\left(\{\mathbf{E}\}^{\text {memb }}+\zeta\{\mathbf{E}\}^{\text {bend }}\right) d \zeta d \bar{\Omega}_{0}
\end{aligned}
$$

In our work we have used a simplified form of Eq. (59) adequate for material linearity problems, where the virtual work of membrane and bending effects is decoupled as

$$
\begin{aligned}
\delta \mathcal{W}^{\text {int }}= & \int_{\bar{\Omega}_{0}} \int_{-\frac{h}{2}}^{\frac{h}{2}} \delta\{\mathbf{E}\}^{\text {memb }} \cdot[\mathbf{C}]\{\mathbf{E}\}^{\text {memb }} d \zeta d \bar{\Omega}_{0} \\
& +\int_{\Omega_{0}} \int_{-\frac{h}{2}}^{\frac{h}{2}} \zeta^{2} \delta\{\mathbb{E}\}^{\text {bend }} \cdot[\mathbb{C}]\{\mathbb{E}\}^{\text {bend }} d \zeta d \bar{\Omega}_{0}
\end{aligned}
$$

The decoupled form of Eq. (60) has been applied to wide range of geometrically nonlinear problems with excellent results, as compared with other existing shell formulations.

In the following a linear material model is used. Integra-

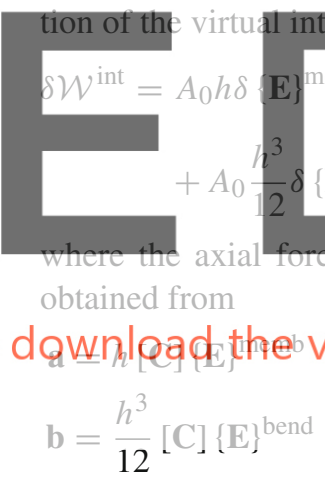

Finally the internal forces for the BST element are computed by

$\mathbf{f}^{\text {int }}=A_{0}\left[\mathbf{B}^{T}\right]^{\text {memb }} \mathbf{a}+A_{0}\left[\mathbf{B}^{T}\right]^{\text {bend }} \mathbf{b}$

The semi-discrete dynamics equations of motion to be solved are given by

$\mathbf{f}^{\text {int }}\left(\mathbf{u}_{n+1}\right)+\mathbf{M} \ddot{u}_{n+1}=\mathbf{f}^{\text {ext }}\left(\mathbf{u}_{n+1}\right)$

where $\mathbf{M}$ is the mass matrix [22].

The acceleration vector $\ddot{\mathbf{u}}_{n+1}$ has to be integrated in time to solve the algebraic equations for $\mathbf{u}_{n+1}$ from the secondorder differential equations. Among the several numerical integration methods available to integrate second order equations, the most popular in structural dynamics is the Newmark scheme. A drawback of the Newmark integrator is the tendency for high noise to persist in the solution. Therefore other integration schemes are utilized with minor modifications as the Generalized- $\alpha$ technique, which is used in this work. 
Fig. 11 Finite element meshes used for the analysis
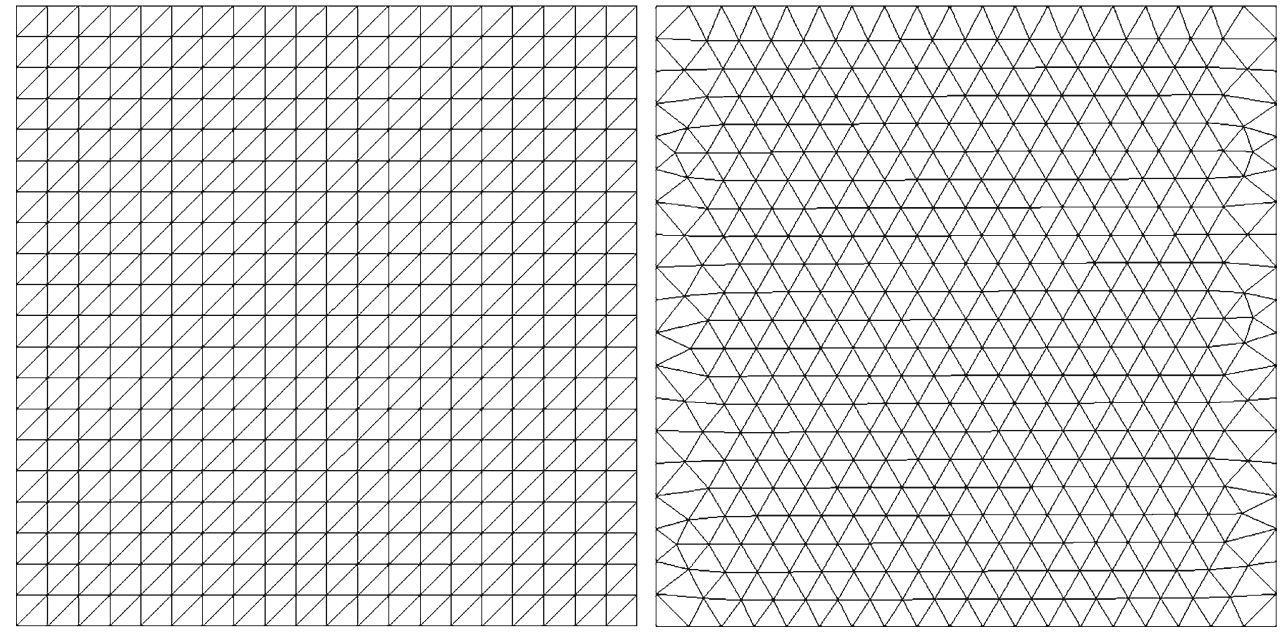

The solution for each external load increment is found using an implicit scheme and the Newton-Raphson iterative method, as described in $[11,12]$. Details of the solution algorithm used in this work can be found in [23].

We finally recall that the results triangular element has the three displacements for each node as the only unknowns.
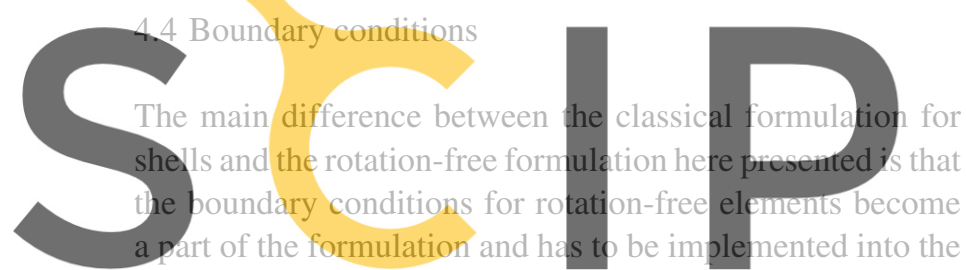

finite element code when building up the bending matrix. In this work the boundary conditions are treated as described
Register for fręat fitps//WWW.scipedia.com to nodes, while the unstructured mesh has 816 elements and 447 nodes. A variable uniform load $q$ is applied, with values from 0.00 to $0.04 \mathrm{~Pa}$. The solution obtained with both meshes was basically identical. The non-dimensional solution of the problem for the structured mesh is plotted in Fig. 12. The transversal displacement $w$ at the central point of the plate is normalized by the thickness $h$, while the load $q$ is normalized
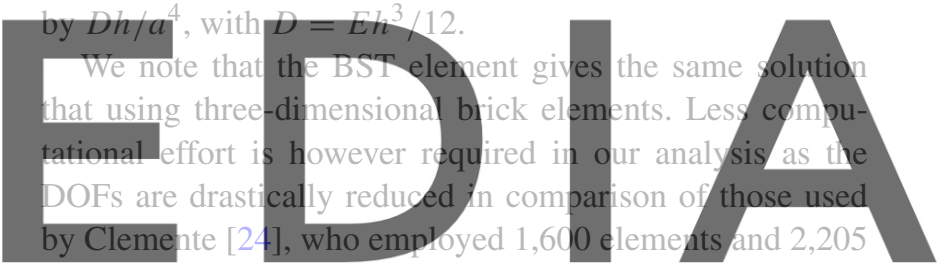

nodes with four layers over the thickness. Figure 12 also shows the importance of accounting for geometrically nondimpload the versign without the watermark

\section{Examples}

\subsection{Nonlinear clamped plate}

Isotropic and orthotropic clamped plates with uniform load are analyzed. The analysis is assumed to be geometrically nonlinear, as presented in Zienkiewicz and Taylor [22] and Clemente [24]. For the isotropic case, the same geometry and material properties of [24] are used here in order to compare the solutions. The only difference is that in [24] 8-noded three-dimensional brick elements are used while in this work BST elements are employed. Orthotropic material properties are $E_{x}=12 \mathrm{~Pa}, E_{y}=6 \mathrm{~Pa}, v_{x y}=0.34$ and $G=$ $3.26 \mathrm{~Pa}$, with $E_{x} v_{y x}=E_{y} v_{x y}$. The side length of the plate is $L=2 a=20 \mathrm{~m}$ with a thickness $h=1 \mathrm{~m}$. For the isotropic case, $E=E_{x}$ and $v=0.0$. Because of the symmetry, only a quarter of the plate is modeled.

The two meshes used for the isotropic analysis are shown in Fig. 11. The structured mesh has 800 elements and 441

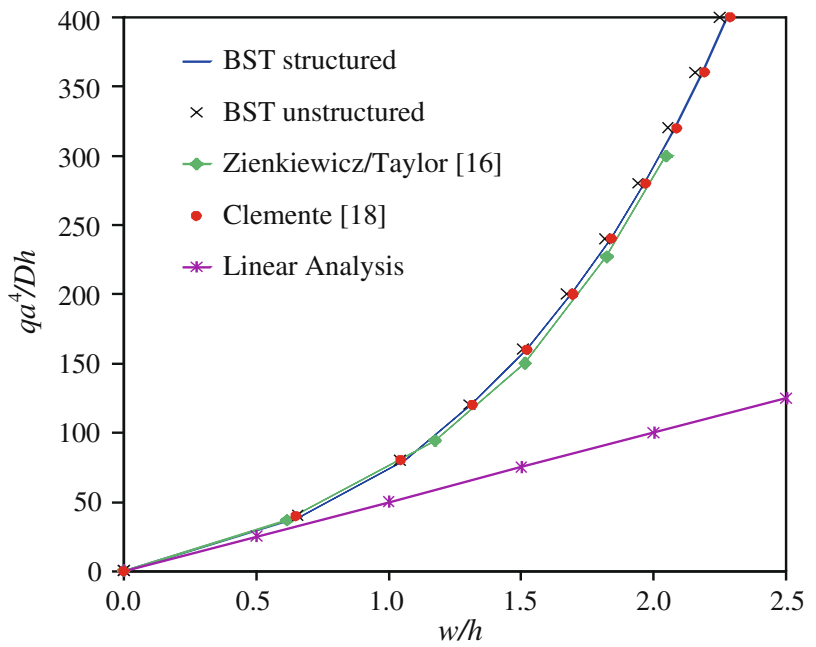

Fig. 12 Central plate displacement versus the uniform load for isotropic material 


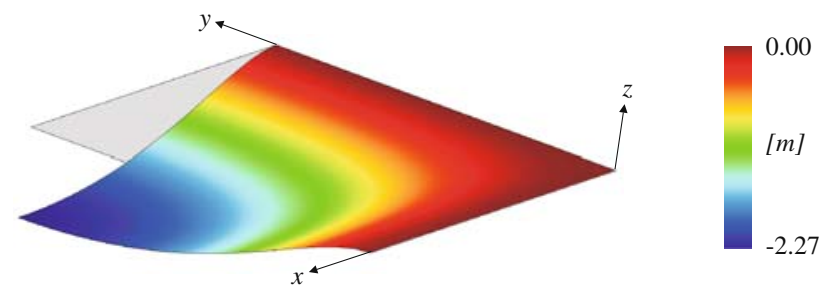

Fig. 13 Vertical displacement contours plotted on the deformed shape for $w / h=-2.27$

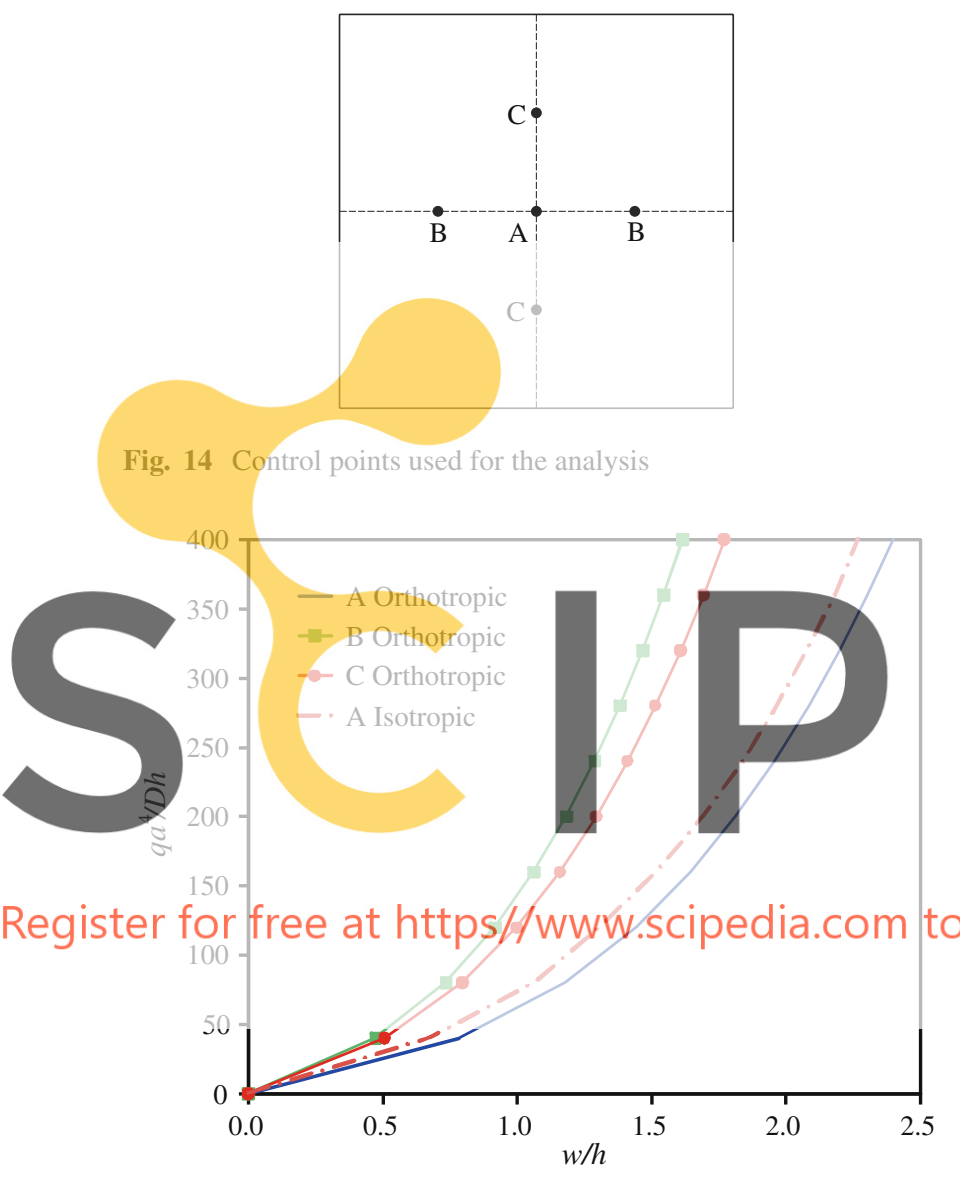

Fig. 15 Displacements of control points $A, B$ and $C$ in the plate of Fig. 13

Figure 13 shows the vertical displacement contours plotted on the deformed shape for a negative value of $q$ and $w / h=-2.27$.

For the orthotropic analysis, a structured mesh with 800 BST elements and 441 nodes is used for the analysis. Figure 14 shows the control points for computing the transversal displacement.

Again, a variable uniform load $q$ is applied to the plate with values from 0.00 to $0.04 \mathrm{~Pa}$, as in the isotropic problem. The non-dimensional solution for the structured mesh is plotted in Fig. 15. The transversal displacements at control points of the plate $w_{A}, w_{B}$ and $w_{C}$ are normalized by the thickness $h$ in the horizontal axis, while the load $q$ is normalized as described previously.

As expected, the displacement of point $B$ is different from that of point $\mathrm{C}$ due to the material orthotropy. For isotropic material the displacements of the control points $\mathrm{B}$ and $\mathrm{C}$ are identical. The maximum deflections are 2.40, 1.61 and $1.77 \mathrm{~m}$ for control points A, B and C respectively in the orthotropic case and $q=0.04 \mathrm{~Pa}$.

\subsection{Hemispherical shell with $18^{\circ}$ hole}

In this example we study an isotropic and orthotropic pinched hemispherical shell based on a popular benchmark problem for linear shell analysis. This problem serves to check the nearly inextensional deformation of a hemispherical shell with an $18^{\circ}$ hole at the top under the action of two inward and two outward forces $90^{\circ}$ apart. Symmetry conditions are used and only one-quarter of the geometry is modeled as shown in Fig. 16.

To illustrate the large deformation capabilities of the BST element, the forces of the linear problem are incremented 100 times to obtain deflections of nearly $60 \%$ of the initial

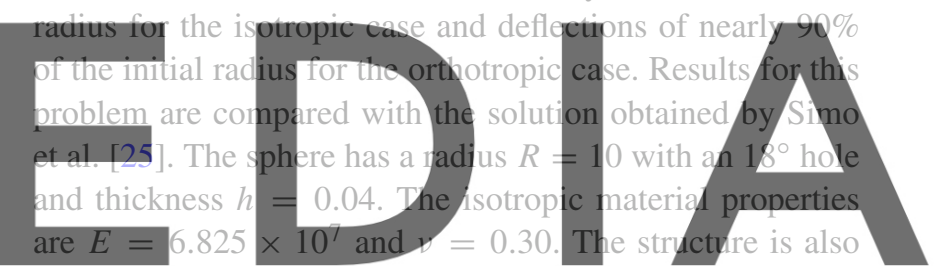

analyzed using three different orthotropic materials with the following properties. OM1, orthotropic material 1 properties: download the versiobswithout the watermark

$5.884 \times 10^{6}$, with $E_{x} v_{y x}=E_{y} v_{x y} . \mathrm{OM} 2: E_{x}=6.825 \times 10^{7}$, $E_{y}=3.413 \times 10^{7}, \nu_{x y}=0.30$ and $G=1.896 \times 10^{7}$ and OM3: $E_{x}=6.825 \times 10^{7}, E_{y}=6.143 \times 10^{7}, v_{x y}=0.30$ and $G=2.518 \times 10^{7}$. Note that OM3 is nearly an isotropic material and the expected solution should be similar as for the isotropic problem. The forces and boundary conditions are shown in Fig. 16, where principal fibers direction are drawn.

The isotropic problem was solved by Simo et al. [25] using a structured mesh of 256 quadrilateral elements with 1,632 DOFs. In this work two meshes are studied for the isotropic problem, a structured mesh of 640 BST elements with 1,036 DOFs, and an unstructured mesh of 640 BST elements with 1,032 DOFs. A summary of the maximum displacements in the $x$ - and $y$-direction is given in Table 2. A plot of the loaddisplacement values is given in Fig. 17.

The complete deformed structured mesh without magnification factor is given in Fig. 18, where the contours of the displacement vector modulus are shown.

The rotation-free BST element has an excellent behavior for this problem involving large displacements. Note the accuracy of the solution with both structured and unstruc- 
Fig. 16 Structured hemispherical shell fiber orientation

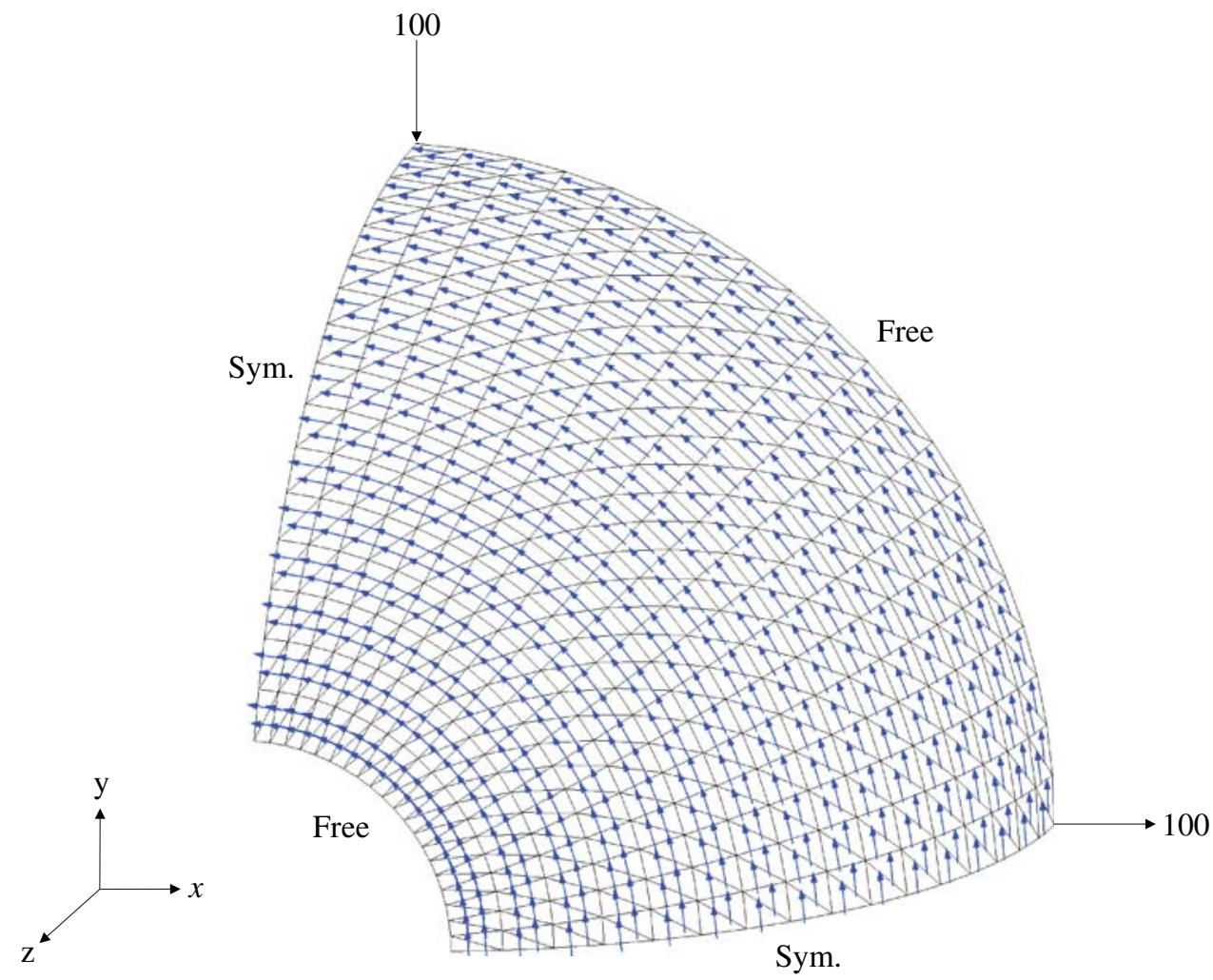

Table 2 Hemispherical shell (maximum displacements)

\begin{tabular}{llllll}
\hline & Elements & DOFs & Mesh & $u$ Disp & $v$ Disp \\
\hline Simo et al. [25] & 256 & 1,632 & Structured & 3.380 & -5.875 \\
Present work & 640 & 1,036 & Structured & 3.319 & -5.878 \\
Present work & 640 & 1,032 & Unstructured & 3.324 & -5.929 \\
\hline
\end{tabular}

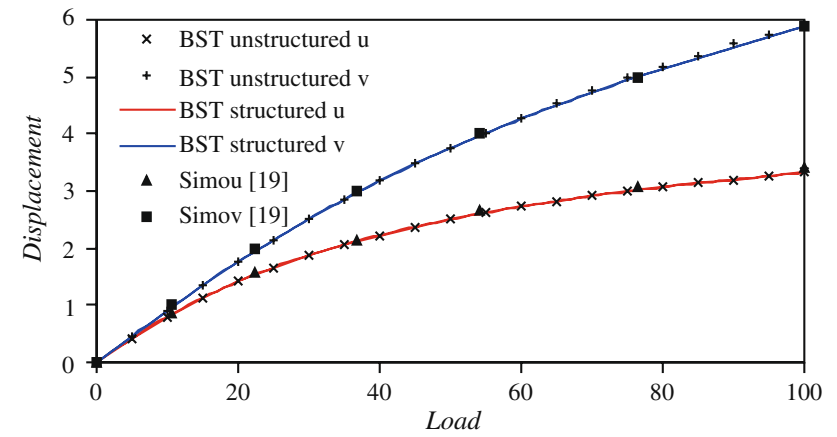

Fig. 17 Load-displacement modulus plot

tured meshes, the former with a uniform element size distribution.

The orthotropic problem is analyzed using a structured mesh of 640 BST elements and 1,036 DOFs. A summary of the maximum displacements in $x$ - and $y$-direction for the different materials is given in Table 3. A plot of the load-displacement curves is shown in Fig. 19.

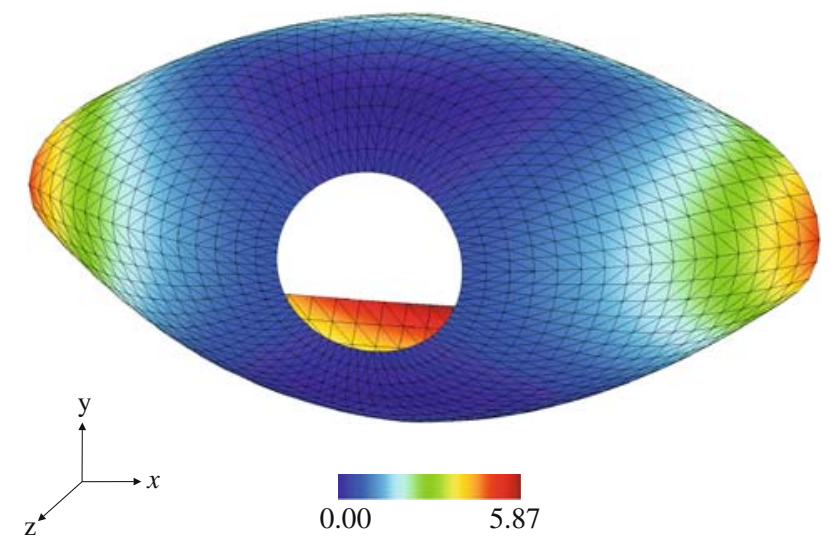

Fig. 18 Deformation without magnification factor. Colors indicate the values of the displacement vector modulus

Table 3 Maximum displacements

\begin{tabular}{lrrr}
\hline & OM1 & OM2 & \multicolumn{1}{c}{ OM3 } \\
\hline$u$-Direction & 3.981 & 3.593 & 3.365 \\
$v$-Direction & -8.913 & -6.999 & -6.054 \\
\hline
\end{tabular}

We recall that OM3 has properties similar to the isotropic case since only one direction of the orthotropic material is $10 \%$ softer in its weakest direction than the isotropic material. The maximum displacements for OM3 are: $u=3.365$ and $v=-6.054$, while the maximum displacements for the 
Fig. 19 Orthotropic hemispherical shell. Load-displacement plots

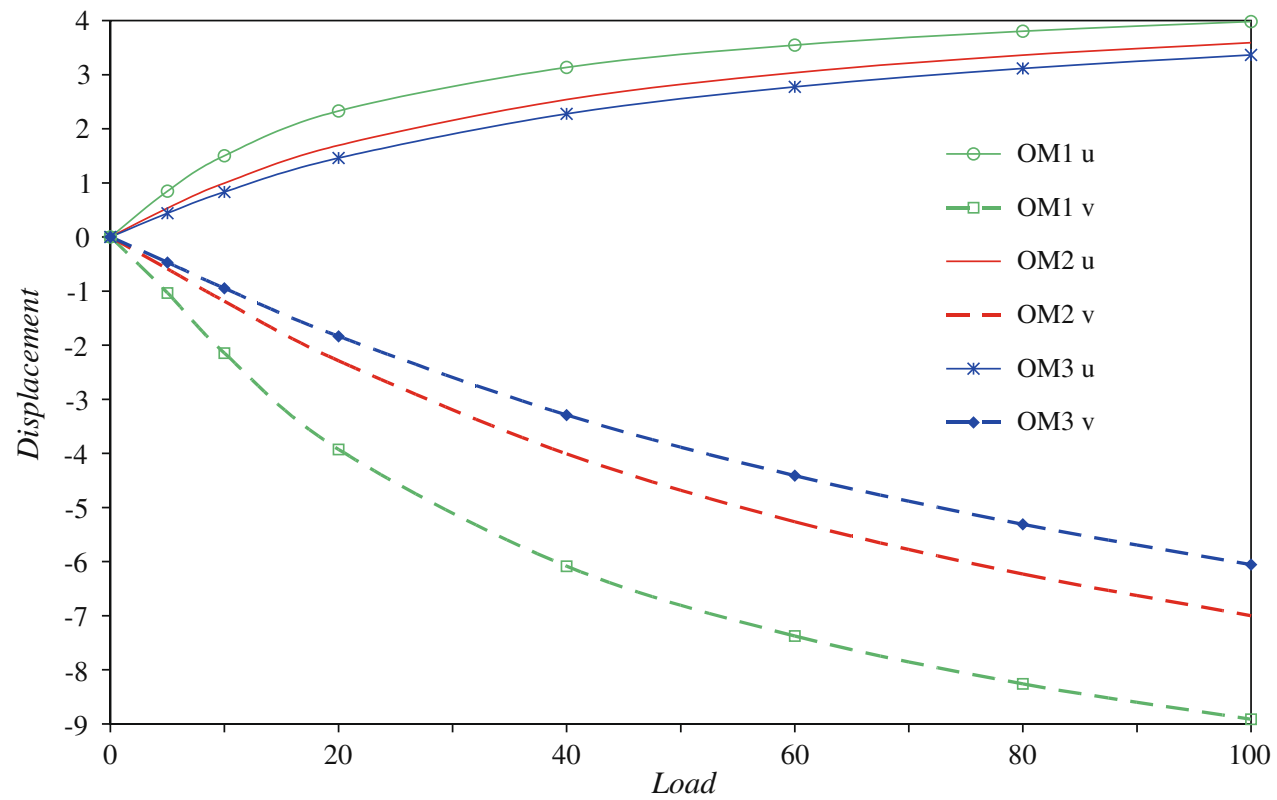

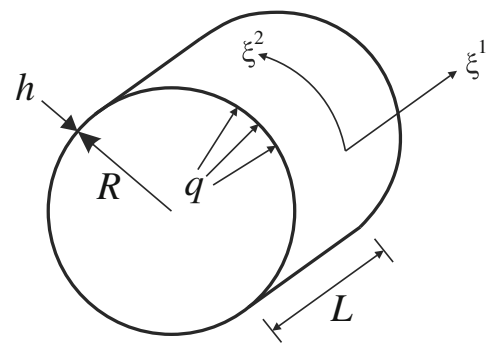

Fig. 20 Geometry for orthotropic clamped cylinder

isotropic material are: $u=3.319$ and $v=-5.878$. Therefore, we can conclude that our analysis for the orthotropic material OM3 is correct, as both solutions are comparable with a maximum difference of $3 \%$.

Orthotropic materials 1 and 2 are 90 and 50\% softer in their weakest direction respectively than the isotropic material, yielding larger displacements than the isotropic case, as shown in Fig. 19.

For OM1, a maximum displacement of $v=-8.913$ is found, yielding a deformation of nearly $90 \%$ of the initial radius. This allow us to conclude that the large deformations capabilities of the BST element are good for orthotropic material.

\subsection{Clamped orthotropic cylinder}

This example is taken from the work of Kreja et al. [26] and Reddy [27]. The problem deals with the nonlinear bending of an orthotropic cylinder clamped at both ends, which geometry is shown in Fig. 20. The cylinder has a radius $R=20.0 \mathrm{in}$, length $L=20.0 \mathrm{in}$ and a thickness $h=1.0 \mathrm{in}$

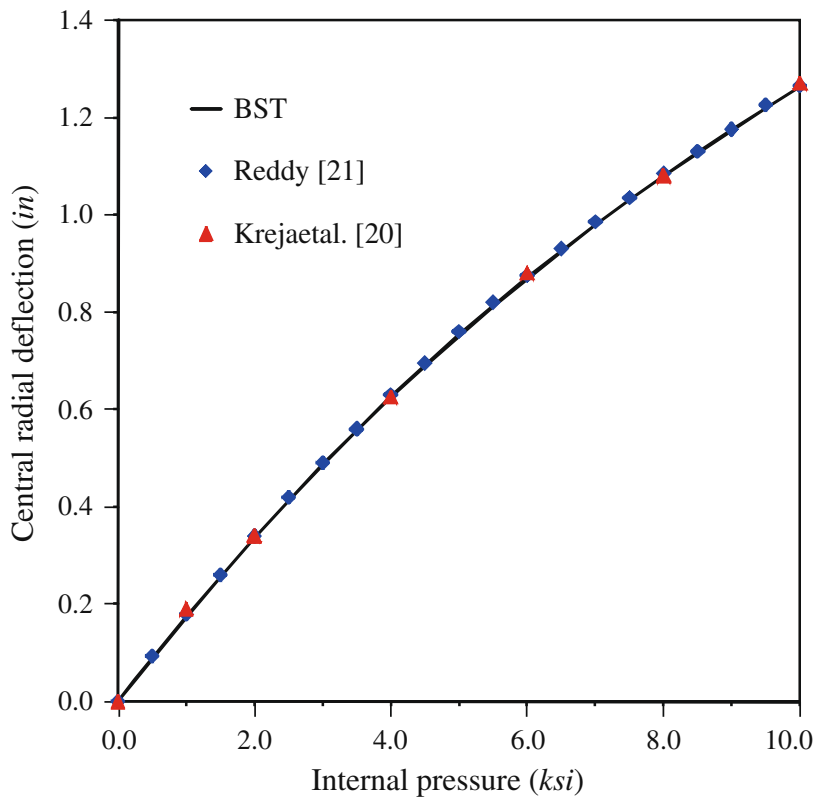

Fig. 21 Center deflection versus internal pressure for orthotropic cylinder

with an internal pressure $q=10.0 \mathrm{ksi}$. The material properties are to those of glass-epoxy fiber-reinforced composite material and are $E_{1}=7,500 \mathrm{ksi}, E_{2}=2,000 \mathrm{ksi}, v_{12}=0.25$ and $G_{12}=1,250 \mathrm{ksi}$ with $E_{1} v_{21}=E_{2} v_{12}$.

In Fig. 21 the central radial deflection versus the applied internal pressure is plotted for various steps. A comparison of these values obtained for the rotation-free orthotropic shell triangle show good agreement with those of Kreja et al. [26] and Reddy [27]. 


\section{Conclusions}

We have presented a rotation-free shell formulation for the geometrically nonlinear analysis of orthotropic thin shell structures using triangular elements. Improvements to the already existing formulation for the rotation-free BST for analysis of orthotropic shell structures have been made using a principal fiber orientation approach.

The idea to use the fiber orientation of the material for the analysis comes from the manufacturing process where a direction for the principal fiber of the material is needed to manufacture the membrane structure. The fiber orientation is mesh dependent and allows the analysis of both isotropic and orthotropic materials. Besides, the fiber orientation allows an adequate postprocessing of the problem as any number of patches that conform the structure can be postprocessed with different local fiber orientations. Another advantage of the fiber orientation approach is that it allows us to perform analysis with orthotropic and composite materials including structural elements having an initial out-of-plane configuration.

\section{References}

1. Ugural AC (1981) Stresses in plates and shells. McGraw-Hill, New York

2. Nay RA, Utku S (1972) An alternative to the finite element method. Var Methods Eng 1(3):63-74

3. Barnes MR (1977) Form finding and analysis of tension space structures by dynamic relaxation. PhD thesis, Department of Civil Engineering, The City University, London

4. Hampshire JK, Topping BHV, Chan HC (1992) Three node triangular elements with one degree of freedom per node. Eng Comput 9:49-62

5. Oñate E, Cervera M (1993) Derivation of thin plate elements with one degree of freedom per node. Eng Comput 10:543-561

6. Brunet M, Sabourin F (1994) Prediction of necking and wrinkles with a simplified shell element in sheet forming. In: Kröplin B (ed) Proceedings of the international conference of metal forming simulation in industry II, pp 27-48

7. Oñate E, Zárate F (2000) Rotation-free triangular plate and shell elements. Int J Numer Methods Eng 47(1-3):557-603

8. Oñate E, Cendoya P, Miquel J (2002) Non linear explicit dynamic analysis of shells using the BST rotation-free triangle. Eng Comput 19(6):662-706

9. Rojek J, Oñate E, Postek E (1998) Application of explicit codes to simulation of sheet and bulk metal forming processes. J Mater Process Technol 80-81:620-627
10. Flores FG, Oñate E (2001) A basic thin shell triangle with only translational dofs for large strain plasticity. Int J Numer Methods Eng 51:57-83

11. Flores FG, Oñate E (2005) Improvements in the membrane behaviour of the three node rotational-free bst shell triangle using an assumed strain approach. Comput Methods Appl Mech Eng 194:907-932

12. Oñate E, Flores FG (2005) Advances in the formulation of the rotation-free basic shell triangle. Comput Methods Appl Mech Eng 194:2406-2443

13. Cirak F, Ortiz M, Schröeder P (2000) Subdivision surfaces: a new paradigm for thin-shell finite element analysis. Int J Numer Methods Eng 47:2039-2072

14. Cirak F, Ortiz M (2001) Fully $c^{1}$-conforming subdivision elements for finite deformation thin-shell analysis. Int J Numer Methods Eng 51:813-833

15. Brunet M, Sabourin F (2006) Analysis of a rotation-free shell element. Int J Numer Methods Eng 66:1483-1510

16. Flores F, Oñate E (2007) A rotation-free shell triangle for the analysis of kinked and branching shells. Int $\mathbf{J}$ Numer Methods Eng 69:1521-1551

17. Linhard J, Wnchner R, Bletzinger KU (2007) Upgrading membranes to shell. The CEG rotation-free shell element and its application in structural analysis. Finite Element Anal Des 44:6374

18. Gärdsback M, Tibert G (2007) A comparisson of rotation-free shell element for unstructured meshes. Comput Methods Appl Mech Eng 196:5001-5015

19. Simo JC, Rifai MS, Fox DD (1990) On a stress resultant geometrically exact shell model. Part IV: variable thickness shells with through-the-time-thickness stretching. Comput Methods Appl Mech Eng 81:91-126

20. Büchter N, Ramm E, Roehl D (1994) Three-dimensional extension of nonlinear shell formulation based on the enhanced assumed strain concept. Int J Numer Methods Eng 37:2551-2568

21. Pimenta PM, Campello EMB, Wriggers P (2004) A fully nonlinear multi-parameter shell model with thickness variation and a triangular shell finite element. Comput Mech 34:181-193

22. Zienkiewicz OC, Taylor RL (2005) The finite element method for solid and structural mechanics, 6th edn. Elsevier, Amsterdam

23. Valdés JG (2007) Nonlinear analysis of orthotropic membrane and shell structures including fluid-structure interaction. $\mathrm{PhD}$ thesis, Technical University of Catalunya. http://www.tdcat.cesca.es/ TDX-1126107-193535

24. Clemente R (2007) Structural analysis of historical buildings with localized crack models. PhD thesis (in spanish), Technical University of Catalunya (UPC)

25. Simo JC, Fox DD, Rifai MS (1990) On a stress resultant geometrically exact shell model. Part III: Computational aspects of the nonlinear theory. Comput Methods Appl Mech Eng 79:21-70

26. Kreja I, Schmidt R, Reddy JN (1997) Finite elements based on a first-order shear deformation moderate rotation theory with applications to the analysis of composite structures. Int $\mathrm{J}$ Nonlinear Mech 32(6):1123-1142

27. Reddy JN (2004) An introduction to nonlinear finite element analysis. Oxford University Press, Oxford 